\title{
THE NATURE OF THE COLD PRESSOR TEST AND ITS SIGNIFICANCE IN RELATION TO NEUROGENIC AND HUMORAL MECHANISMS IN HYPERTENSION ${ }^{1}$
}

\author{
BY MORTON F. REISER AND EUGENE B. FERRIS, JR. \\ (From the Department of Internal Medicine, University of Cincinnati College of Medicine \\ and Cincinnati General Hospital, Cincinnati)
}

(Received for publication August 20, 1947)

\section{INTRODUCTION}

Recent emphasis on the use of partial sympathectomy in the treatment of essential hypertension has focused attention on the "neurogenic component" in this disease. That the autonomic nervous system plays a role cannot be questioned; however, the physiologic basis for most operative procedures in hypertension has not been clearly established. Humoral pressor mechanisms have also been emphasized as being of primary significance in its pathogenesis but, likewise, their role in clinical hypertension is not clear.

It becomes, then, a matter of great importance for the further understanding of this disease to develop methods which will make it possible to identify at any given time the presence, extent, and character of autonomic vasomotor activity. Such methods, in addition to providing information regarding the nature of neurogenic mechanisms, should at the same time help to evaluate nonnervous factors and their inter-relationships with vegetative nervous control. In a study of blood pressure levels (1) utilizing differential methods for partial and complete sympathetic denervation, it was found that in a majority of cases of hypertension there apparently is a direct quantitative relationship between the level of the blood pressure and the anatomical extent of the arteriolar bed under active vasomotor control. The implications with regard to reduction of blood pressure by partial sympathectomy were discussed.

The present study concerns itself with the effector mechanism of the cold pressor response in hypertension, utilizing varying degrees of sympathetic block induced by spinal anesthesia and tetraethyl ammonium chloride (TEAC). Although, as pointed out elsewhere (2), the clinical value of

1 The work described in this paper was aided in part by a grant from the Psychosomatic Research Fund of the National Committee for Mental Hygiene, Inc. this test is limited, the evidence to be presented suggests that its utilization together wtih other procedures may be useful in evaluating the relative importance of neurogenic and humoral components in hypertension.

\section{MATERIAL AND METHODS}

Twenty hypertensive patients are included in this study, 15 having benign essential hypertension, 2, malignant hypertension, 2, polycystic kidneys and 1 , acute glomerulonephritis. TEAC 2 in doses of 3 to $5 \mathrm{cc}$. (300 to 500 mgm.) administered intravenously was used to induce total sympathetic block (3). Partial sympathetic block was induced by means of spinal anesthesia to varying spinal levels and in 1 additional case, by partial surgical sympathectomy. The cold pressor test (4) was performed in the usual manner, blood pressure being measured after the $1 / 2$ - and 1-minute intervals of immersion of the hand in water at $4^{\circ} \mathrm{C}$. (the "cold minute" response). In addition blood pressure measurements were continued after removal of the hand from cold water in order to detect delayed pressor effects.

The procedure was to obtain a control cold pressor response after the subject had rested for $1 / 2$ hour and after pre-test blood pressure levels had been determined at 1-minute intervals for at least $\mathbf{5}$ minutes or until a stable base line level was obtained. Soon after the control cold pressor test, TEAC was administered to 20 cases and the cold pressor test repeated during the second and third minutes after injection of TEAC, blood pressures being measured frequently throughout the period of TEAC action. Because of the rapid change in blood pressure in those subjects who showed a depressor response to TEAC, the "cold minute" period sometimes coincided with either the downswing or upswing of the TEAC response which in these and other patients has exhibited a consistent pattern.

In 8 cases the cold pressor response was again tested after high spinal anesthesia and in 5 , it was repeated as the anesthesia receded to lower levels. In 5 cases, after the response of the cold pressor test to spinal anesthesia was determined, the subjects were given TEAC and the cold pressor test again repeated in the usual manner.

In an additional case of essential hypertension not included in the above series, 2 control cold pressor tests

2 Etamon chloride supplied by Parke, Davis and Company through the courtesy of Dr. E. C. Vonder Heide. 


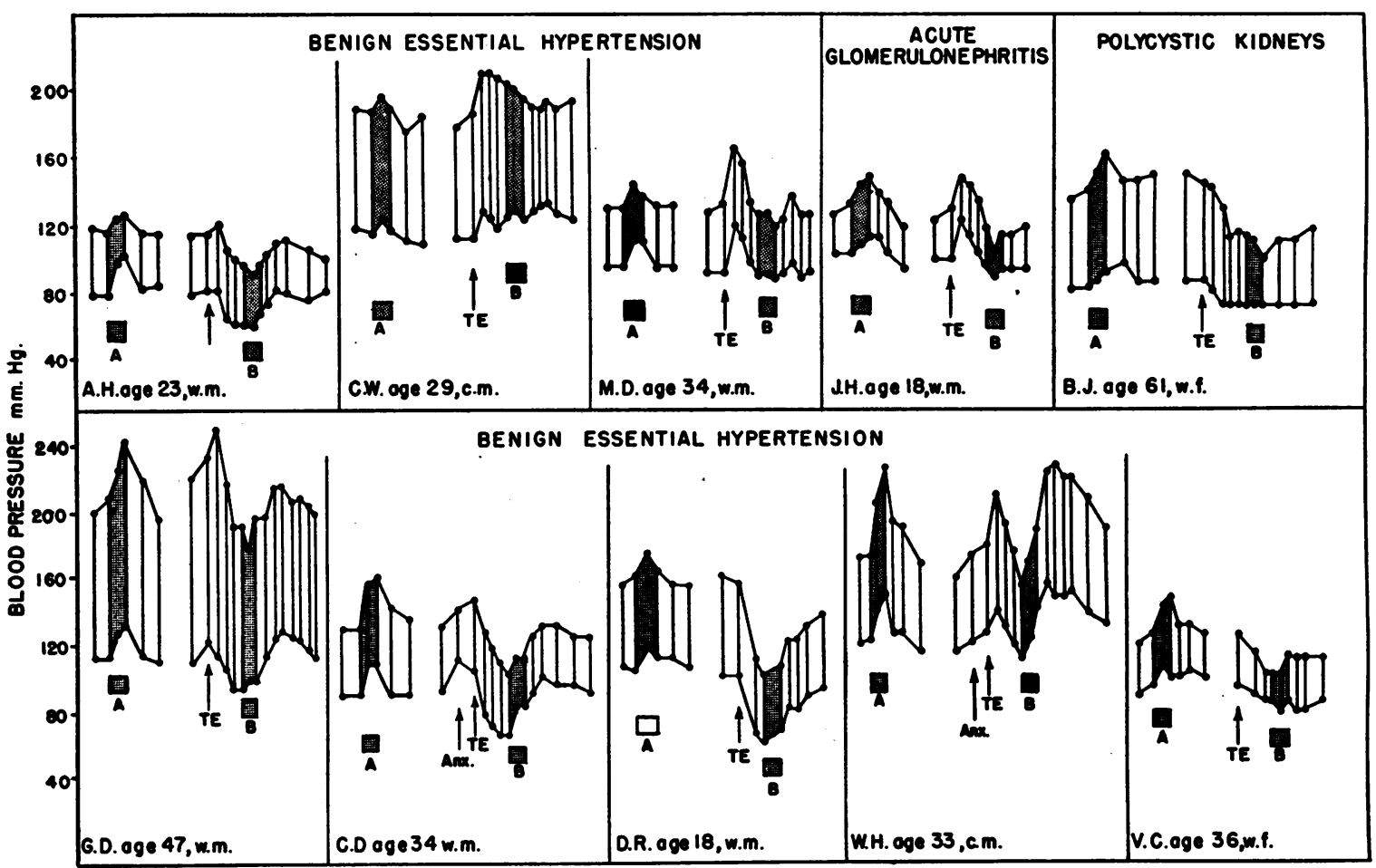

Fig. 1. The Effect on the Cold Pressor Response of Pharmacologic Autonomic Blockade Produced by the Intravenous Administration of TEAC

The short intervals represent 30 -second readings, the long intervals minute readings of the blood pressure. The cold minute in each instance is identified by the gray stippling. " $A$ " represents the control cold pressor reaction; " $B$ " the cold pressor reaction during the action of TEAC. Note that in all instances after the administration of TEAC (TE) the cold minute reaction is eliminated and that the cold minute exposure in no way alters the curve of the TEAC reaction in those cases where a depressor response is produced by the drug. "Anx." denotes the appearance of overt anxiety. Note the poor depressor response and marked delayed cold pressor rise in patient W. H., who showed marked anxiety prior to injection of TEAC.

were done before sympathectomy and 4 months postoperatively the response was again tested before and during the action of TEAC.

\section{RESULTS}

A. Effects of blockade of the autonomic ganglia by the intravenous administration of TEAC:

(1) Upon the level of the blood pressure: In 14 cases (70 per cent) the blood pressure was significantly lowered, to normal in 10 , near normal in 1 and to intermediate levels in 3 . The lowest values were usually obtained between the second and third minutes following the injection. Thereafter, the blood pressure showed a steady upward trend. Six patients ( 30 per cent) failed to obtain depressor responses and it should be noted that each of these responded to TEAC with a definite immediate rise in blood pressure which reached its peak at 30 seconds following the injection and lasted 1 to $1 \frac{1}{2}$ minutes. $^{3}$ This rise should be distinguished from the transient rise seen in patient E. H. (Figure 2) which was due to anxiety and pain connected with the venepuncture. Within 30 seconds it was followed by a typical depressor response to the injection of TEAC.

(2) Upon the cold pressor response: Two phases of blood pressure response to cold were noted. First, the response which occurred during the minute of exposure to cold ("cold minute"), the classical cold pressor response, and second,

${ }^{3}$ In this particular series this immediate rise in blood pressure happened to occur in all of the patients who failed to obtain depressor responses. This is not invariably the case, however, as in other patients who have failed to obtain depressor responses to TEAC, this immediate rise has not been noted. 


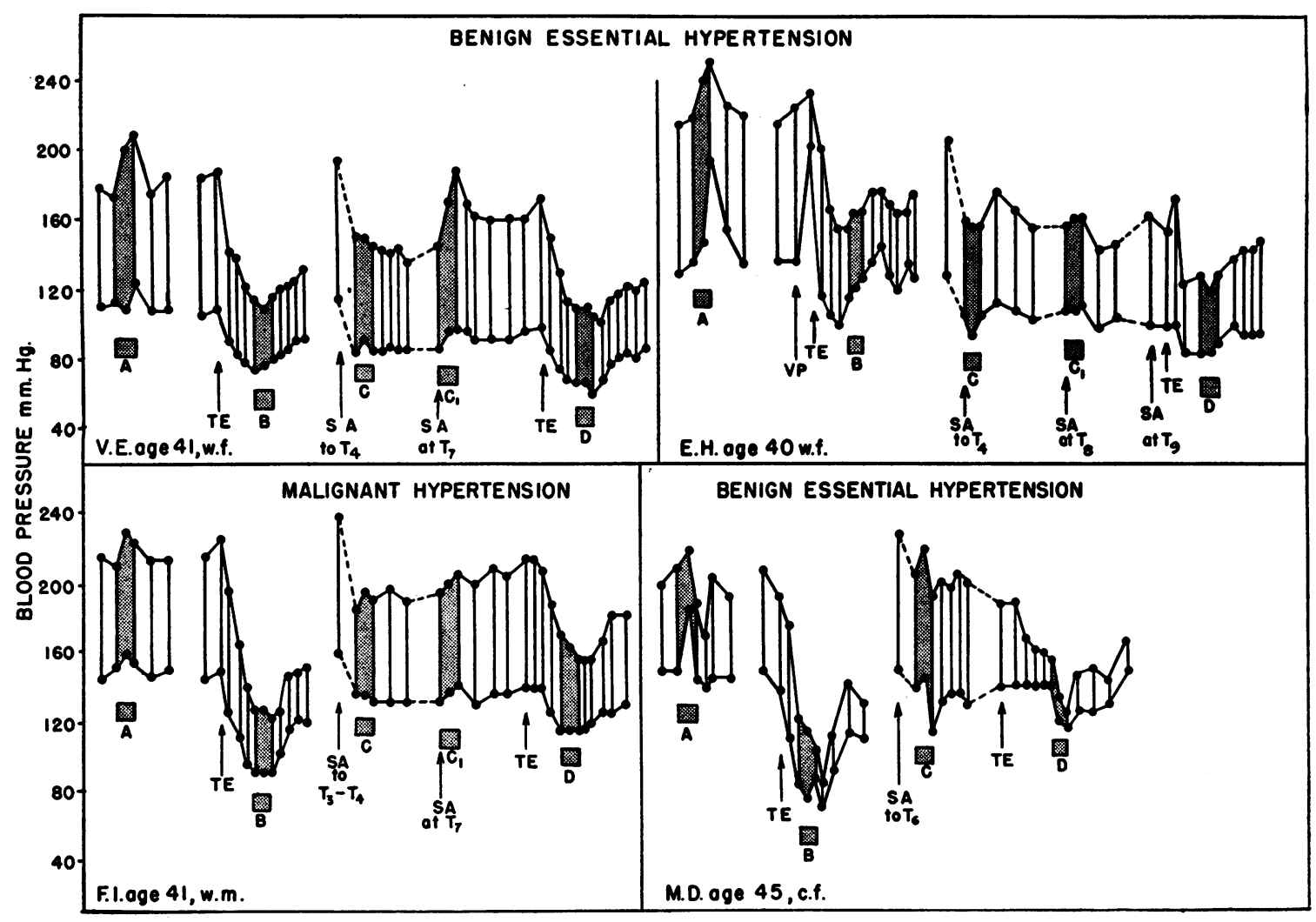

Fig. 2. The Effect on the Cold Pressor Response of Varying Degrees of Autonomic Blockade

The time intervals are denoted in the same manner as in Figure 1. Cold minute exposures are denoted as follows: A-Control. B-during the action of TEAC. C-under high spinal anesthesia (SA). $\mathrm{C}_{1}-$ under spinal anesthesia at lower level. D-after administration of TEAC (TE) during the period of spinal anesthesia. VP denotes venepuncture. Note the complete abolition of the cold minute response during the period of TEAC effect and the varying degrees of dampening of this reaction at different levels of spinal anesthesia. Fall in blood pressure in response to cold exposure under conditions of autonomic blockade seen in patient M.D. is characterized by narrowing of the pulse pressure and is felt to be a manifestation of venous pooling.

delayed pressor responses which occurred after the hand had been removed from cold water.

In all cases TEAC abolished the cold minute pressor response irrespective of the type of hypertension, the degree of depressor effect of the drug, or of the type of control cold pressor reaction. In those cases in which the blood pressure fell in response to TEAC the cold minute exposure did not significantly influence the character of the TEAC response, whether the exposure occurred during the downswing, floor, or upswing of the blood pressure curve (Figures 1, 2, and 3).

In 1 case in which the cold minute occurred during the downswing of the TEAC blood pressure reaction, a very sharp transient rise in pressure occurred immediately after removal of the hand from cold water (Figure 4). This patient had had a delayed recovery period during his control cold pressor test and reacted with a great deal of anxiety and protest to the discomfort of the cold pain. Similar but much less marked delayed transient elevations in pressure following the cold pressor test performed under these conditions of autonomic block were seen in 11 other patients. There was no correlation between the occurrence of this delayed phenomenon and the patient's reaction to the drug in terms of modification of blood pressure level.

$B$. Comparison of the effects on the cold pressor response of total autonomic blockade (TEAC) with those of partial sympathetic denervation (high spinal anesthesia). (Table I).

A level of T4 or slightly above was attained in 7 instances. In all but 1 the cold pressor response 
was eliminated at this level. In 5 of these the response was again tested and found to be active (partially in 4, completely in 1) when the anesthesia had receded to lower levels of $\mathrm{T} 7$ to $\mathrm{T} 8$.

In 5 cases which showed an active cold pressor response at levels of $\mathrm{T} 6$ to $\mathrm{T} 8$ the response was again eliminated by the intravenous administration of TEAC during the period of anesthesia (Figure 5).

In the patient tested 4 months after partial sympathectomy (analogous to partial denervation by spinal anesthesia) the cold pressor response was found to have remained fully active. TEAC given intravenously then abolished this response.

\section{DISCUSSION}

Wolf and Hardy have demonstrated that the cold pressor reaction is initiated by a particular type of pain termed "cold pain," and that the afferent limb of the reflex is mediated over nervous pathways (5). The mechanism of the effector phase has remained the subject of speculation.

It has been amply demonstrated that TEAC administered in adequate doses effects a transient autonomic block, and that the site of action is at the autonomic ganglia (3). The action of substances such as adrenalin or angiotonin which act upon the neuro-effector apparatus at a point peripheral to the ganglia is not eliminated by this drug (3). It follows then that phenomena mediated through the autonomic nervous system will be abolished by the action of TEAC whereas effects caused by certain humoral mechanisms will remain. Examination of the results of this study indicates that the effector phase of the cold pressor reaction is neurogenic. In all instances the re-

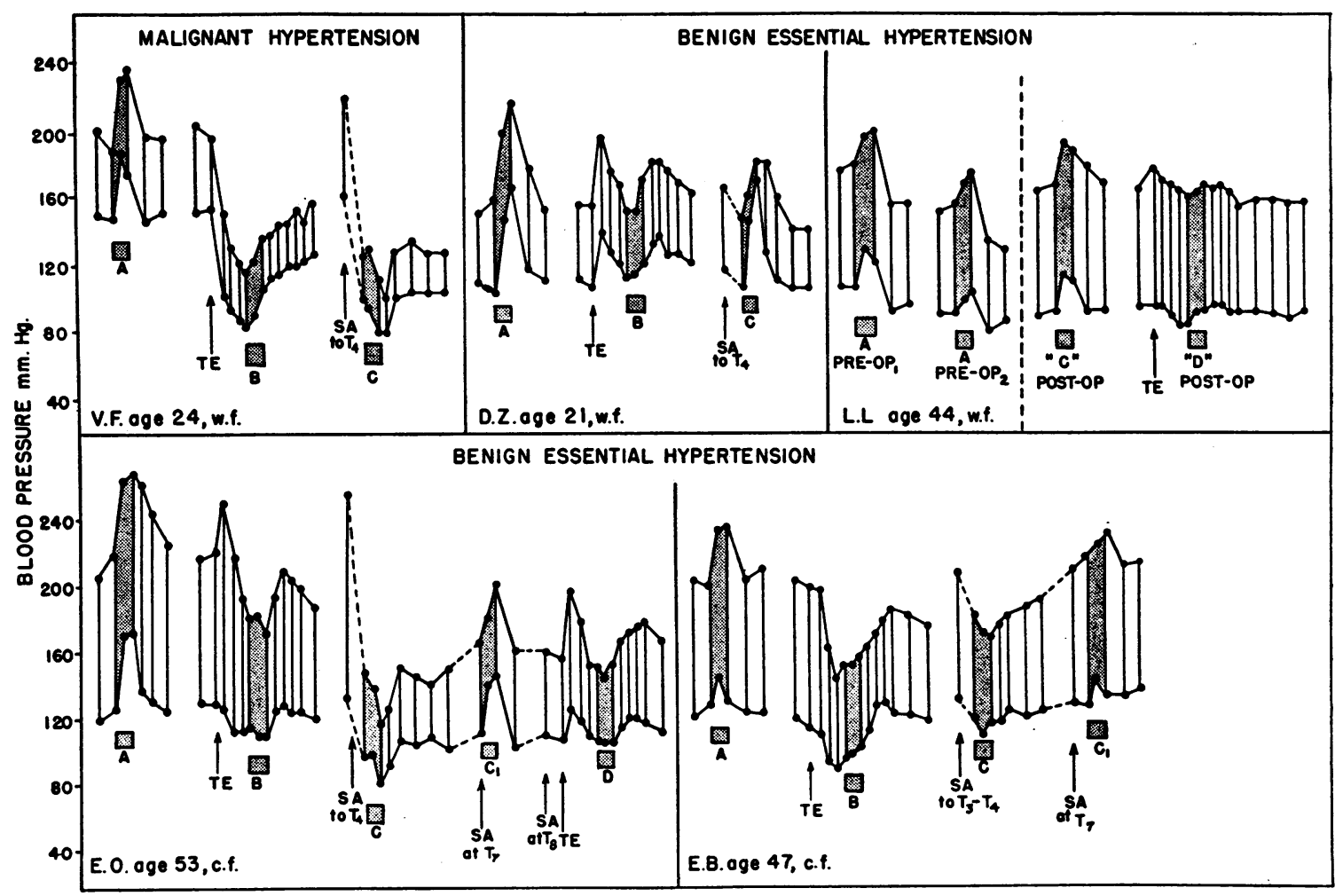

Fig. 3. The Effect on the Cold Pressor Response of Varying Degrees of Autonomic Blockade

The scheme of presentation is the same as that in Figures 1 and 2. Note again the abolition of the cold minute response by TEAC (TE) action and the varying degrees of dampening, of the response at different levels of spinal anesthesia. In patient L. L., "C" (post-operative) designates the cold pressor reaction 4 months after partial sympathectomy (analogous to spinal anesthesia) and "D" (post-operative) represents the effect of TEAC on this post-operative response. Fall in pressure in response to cold exposure during spinal anesthesia in patients V. F. and $E$. O. is felt to be a manifestation of venous pooling. 


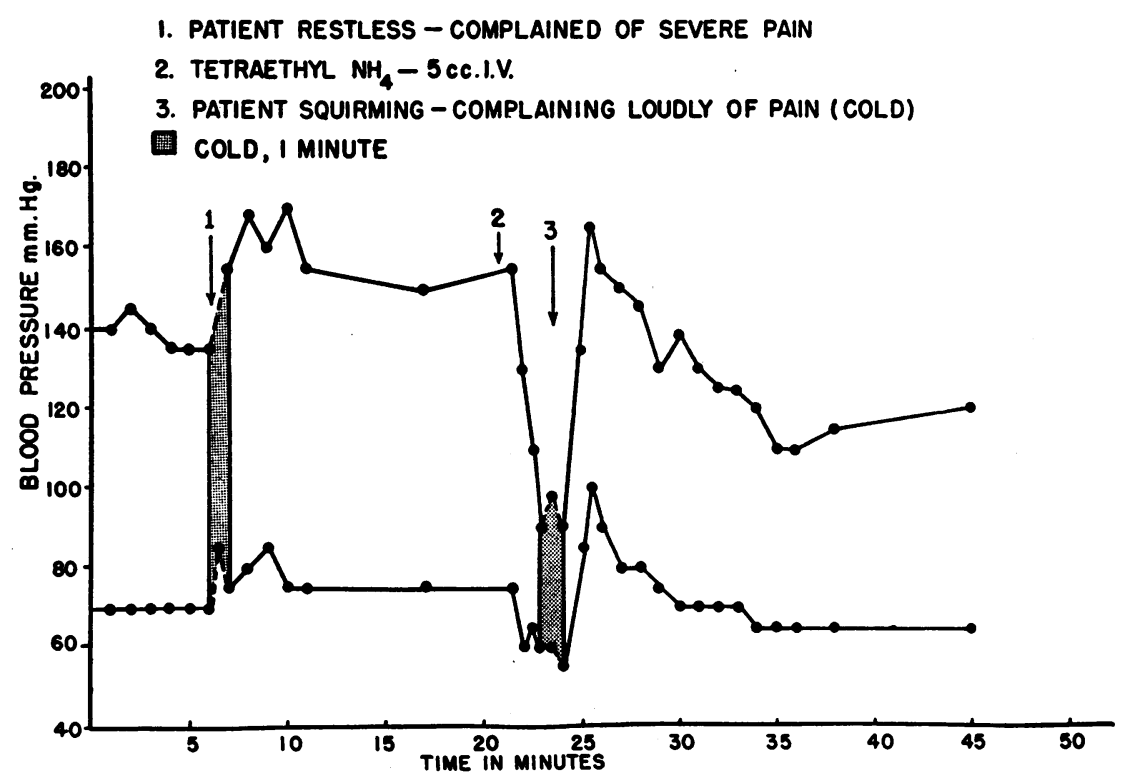

Fig. 4. The Cold Pressor Reaction in a Patient Before and After the Administration of TEAC (TE)

Note the patient's marked subjective response to the experiencing of cold pain, the continuing rise of pressure and delayed recovery after the control cold minute, and the corresponding marked delayed pressor response following cold exposure after TEAC even though the cold minute response was eliminated.

TABLE I

Effect of varying degrees of sympathetic block on cold pressor response

\begin{tabular}{|c|c|c|c|c|c|}
\hline \multirow{2}{*}{ Patient } & \multirow{2}{*}{ Diagnosis* } & \multirow{2}{*}{$\begin{array}{l}\text { Effect on cold pressor } \\
\text { response of TEAC alone }\end{array}$} & \multicolumn{2}{|c|}{ Spinal anesthesia } & \multirow{2}{*}{$\begin{array}{l}\text { Effect on cold pressor } \\
\text { response of adding TEAC } \\
\text { to spinal anesthesia } \\
\text { (at a level at which the } \\
\text { response is still active) }\end{array}$} \\
\hline & & & Level & $\begin{array}{l}\text { Effect on cold } \\
\text { pressor response }\end{array}$ & \\
\hline 1. V.F. & M. H. & Eliminated & $\mathrm{T} 4$ & Eliminated & \\
\hline 2. F. I. & M. H. & Eliminated & $\begin{array}{l}\text { T3-4 } \\
\text { T7 }\end{array}$ & $\begin{array}{l}\text { Eliminated } \\
\text { Dampened }\end{array}$ & Eliminated \\
\hline 3. M. D. & B. H. & Eliminated & T6 & Dampened & Eliminated \\
\hline 4. V. E. & B. $\mathrm{H}$. & Eliminated & $\begin{array}{l}\mathrm{T} 4 \\
\mathrm{~T} 7\end{array}$ & $\begin{array}{l}\text { Eliminated } \\
\text { Unaffected }\end{array}$ & Eliminated \\
\hline 5. E. H. & B. H. & Eliminated & $\begin{array}{l}\mathrm{T} 4 \\
\mathrm{~T} 8\end{array}$ & $\begin{array}{l}\text { Eliminated } \\
\text { Dampened }\end{array}$ & Eliminated \\
\hline 6. D. Z. & B. $\mathrm{H}$. & Eliminated & $\mathrm{T} 4$ & Unaffected & \\
\hline 7. E. O. & B. H. & Eliminated & $\begin{array}{l}\mathrm{T} 4 \\
\mathrm{~T} 7\end{array}$ & $\begin{array}{l}\text { Eliminated } \\
\text { Dampened }\end{array}$ & Eliminated \\
\hline 8. E. B. & B. $\mathrm{H}$. & Eliminated & $\begin{array}{l}\text { T3-4 } \\
\text { T7 }\end{array}$ & $\begin{array}{l}\text { Eliminated } \\
\text { Dampened }\end{array}$ & \\
\hline
\end{tabular}

* B. H.-Benign essential hypertension.

M. H.-Malignant hypertension. 


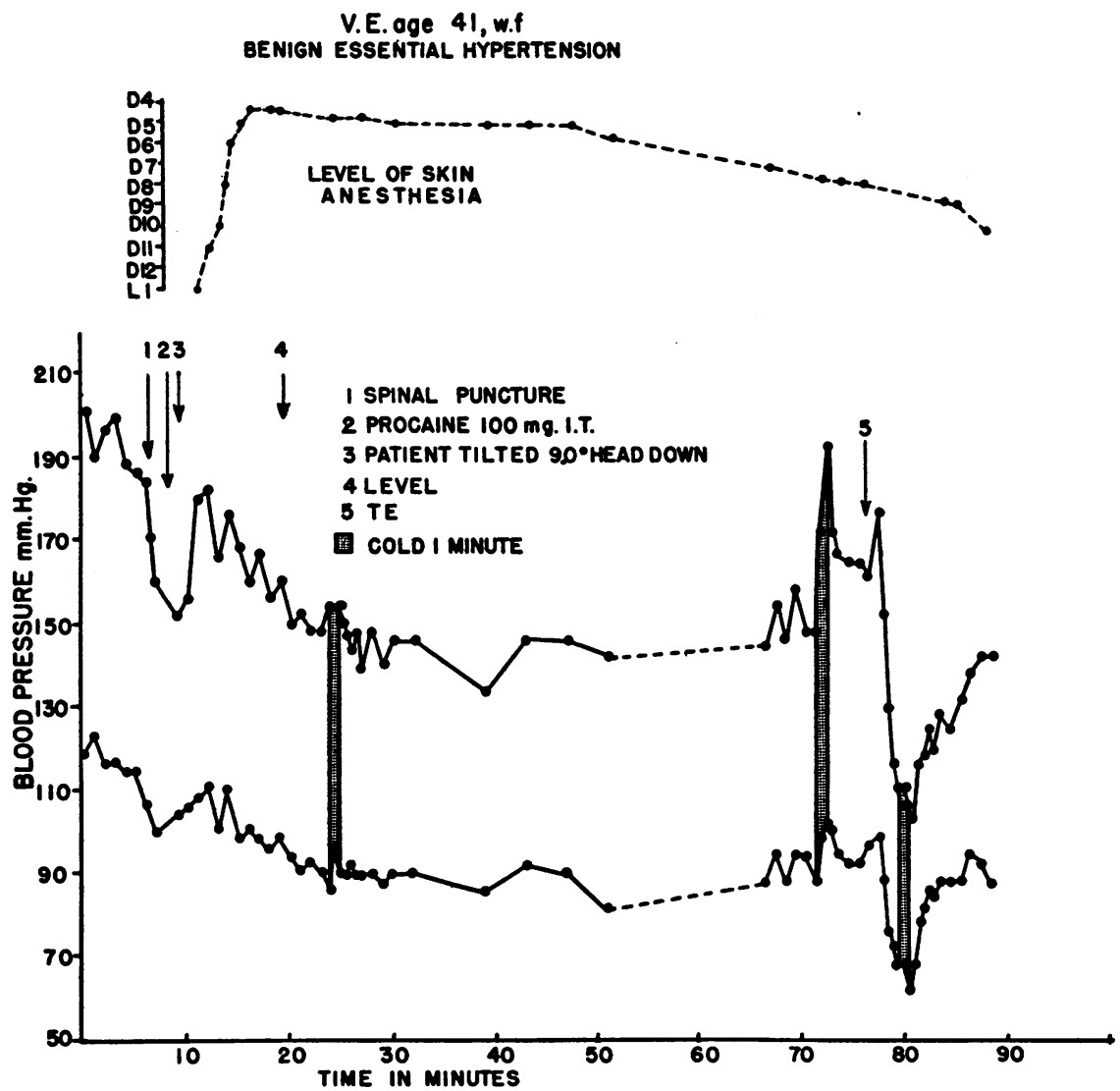

Fig. 5. Example Illustrating the Effect of Varying Degrees of Autonomic Blockade on the Cold Pressor Response

Note that when the level of spinal anesthesia is at D4-5 the cold pressor response is eliminated. At a lower level of D7 the cold pressor response is fully active. After the administration of TEAC (TE) at this level the response is again abolished. This would seem to indicate that in this particular patient clinically significant cold pressor reactivity emanates from the spinal cord between segments D4 and D7.

sponse was abolished by autonomic blockade. ${ }^{4}$ In those instances where the cold minute exposure coincided with the upswing of the TEAC response, it can be seen that the small rises in blood pressure during the cold minute were actually part of the

\footnotetext{
4 An additional patient studied after the completion of this series, showed a very vigorous control cold pressor reaction which was not affected by spinal anesthesia to T6 or by the injection of $4 \mathrm{cc}$. (400 mgm.) of TEAC. Administration of $8 \mathrm{cc}$. ( $800 \mathrm{mgm}$.) of TEAC completely abolished the cold pressor response. This would indicate (as might be expected ) that the size of the fully effective dose of TEAC may vary among different patients. The effect on the cold pressor reaction might well be used to gauge the effectiveness of dosage in producing autonomic blockade in individual patients.
}

recovery phase of the blood pressure following the evanescent peak action of the drug. In addition such cold minute patterns differ from those of the cold pressor reaction in that there is no subsequent return of pressure to control pre-test level.

It has been pointed out elsewhere (1) that in hypertensives who demonstrate an active neurogenic component the level of the blood pressure tends to vary directly with the anatomical extent of the arteriolar bed under active vasomotor control. The present results demonstrate that the same concept applies to variations in pressor reactivity in a given individual. It will be noted that sympathetic block (TEAC) abolished the cold pressor reaction. Release of varying portions 
of the arteriolar bed from active vasomotor control (spinal anesthesia of varying extent) produces dampening of the response, the degree of which tends to vary with the extent of "denervation." As in the case of the effect on the level of the blood pressure, although the relationship tends to be a direct one, the smallest remaining portion of the arteriolar bed which is capable of exciting clinically significant cold pressor reactivity appears to vary from patient to patient. This probably accounts for the finding that a standard operative procedure (lumbodorsal splanchnicectomy) has produced no uniformity of effect on the pattern of the cold pressor response in a large series of patients (6). The findings in the patient, L. L. (Figure 3), tested after partial sympathectomy, are consistent with this view and suggest that a portion of the sympathetic system large enough to exert clinically significant pressor reactivity was left intact. Although there are not enough determinations in this series to be significant in this respect, the fact that in 6 out of 7 patients, anesthetic levels between T3 and T4 were effective in totally eliminating the response, would suggest that most of the significant cold pressor reactivity is controlled from below this level in the majority of patients.

The demonstration of the neurogenic character of the "cold minute" rise is helpful in leading to an understanding of the delayed cold pressor response seen in exaggerated form in 1 patient ( $E$. H., Figure 4), and to a lesser but significant degree in 11 others. This pressor phenomenon may be humoral in character because it occurs at a time when the sympathetic impulses are blocked at the ganglia, and while the arterioles are still responsive to certain humoral pressor substances. Similarly it also seems likely that the initial rise in blood pressure observed after TEAC in 6 of the patients is humoral in origin.

It is of interest to note that the patient having such a vigorous delayed pressor response exhibited a slow return of his blood pressure to the pre-test baseline during the control cold pressor test. This would suggest that the delayed recovery seen in some patients following the cold pressor test is dependent upon a humoral mechanism in contrast to the neurogenic cold minute phase. In this experiment (Figure 4) the patient exhibited marked anxiety in response to the test situation, and it seems likely that the humoral substance respons- ible for the response under consideration may have been epinephrine. ${ }^{5}$

Likewise, the fact that the cold pressor response was eliminated in those patients who failed to show a depressor response to the administration of TEAC would suggest that this apparent inefficacy of the drug was due to the maintenance of the blood pressure by a humoral agent, rather than to a failure of the drug to produce autonomic paralysis. This concept is further supported by the findings reported elsewhere (7) that when TEAC is given repeatedly the type of blood pressure response may vary a good deal from day to day so that a patient who fails to react with a large depressor response at one time may do so on a different occasion.

\section{SUMMARY AND CONCLUSIONS}

1. Intravenous administration of TEAC to each of 20 patients produced significant lowering of the blood pressure in 14 (70 per cent). Each of the 6 patients ( 30 per cent) who failed to obtain a depressor response exhibited an initial transient rise in blood pressure following the administration of the drug. It is felt that this rise originates in a humoral pressor mechanism.

2. Following the administration of TEAC the cold pressor response was eliminated in all patients tested, indicating that the mechanism of the cold pressor response is a neurogenic one.

3. The evidence suggests that the cold pressor response may be useful in evaluating procedures designed to eliminate autonomic tone in hypertension.

4. Comparison of the cold pressor responses under varying degrees of spinal anesthesia with those under total autonomic blockade (TEAC) reveals a relationship between the extent of the arteriolar bed under active vasomotor control and the degree of reactivity to the cold pressor test.

5. Delayed cold pressor response, which occurred in 12 out of 20 patients under autonomic

5 It may be argued that this is unlikely since the adrenal medulla is innervated by sympathetic fibers whose action should be blocked by the effect of TEAC. Actually the cells of the adrenal medulla are unique in that they are innervated by preganglionic fibers, being themselves analogous to the cells of the sympathetic ganglia. TEAC may not block this connection as it does those at the ganglia. To our knowledge no information on this point is available. 
blockade produced by TEAC, can best be explained by a humoral meclanism. It is suggested that the delayed recovery phase of the cold pressor response seen in some patients may be a manifestation of this phenomenon.

6. It is suggested that failure in some cases to obtain depressor responses to TEAC may be due to the presence of a humoral pressor substance rather than to failure of the drug to produce a satisfactory autonomic blockade.

7. The data suggest that both humoral and neurogenic (autonomic) mechanisms may interact as factors in hypertension.

\section{ACKNOWLEDGMENTS}

The authors would like to express their appreciation to Miss Mary A. Costello, Chief Anesthetist of the Department of Surgery, University of Cincinnati, and her assistants for their aid in administering the spinal anesthetics. The assistance of Mrs. Jane K. Friedlander and Dr. Joseph E. Levinson is also gratefully acknowledged.

\section{BIBLIOGRAPHY}

1. Reiser, M. F., and Ferris, E. B., Jr., Evaluation of neurogenic control of blood pressure in hypertension with tetracthyl ammonium chloride and spinal anesthesia. To be published.
2. Reiser, M. F., and Ferris, E. B., Jr., Clinical and experimental observations on the lability and range of blood pressure in hypertension with special reference to the evaluation of various pressor and depressor tests. To be published.

3. Lyons, R. H., Moe, G. K., Neligh, R. B., Hoobler, S. W., Campbell, K. N., Berry, R. L., and Rennich, B. R., The effects of blockade of the autonomic ganglia in man with tetraethyl ammonium. Preliminary observations on its clinical application. Am. J. M. Sc., 1947, 213, 315.

4. Hines, E. A., and Brown, G. E., A standard stimulus for measuring vasomotor reactions: Its application in the study of hypertension. Proc. Staff Meeting Mayo Clinic, 1932, 7, 332.

5. Wolf, S., and Hardy, J. D., Studies on pain. Observations on pain due to local cooling and on factors involved in the "Cold Pressor" effect. J. Clin. Invest., 1941, 20, 521.

6. Smithwick, R. H., Surgical treatment of hypertension. The effect of radical (lumbodorsal) splanchnicectomy on the hypertensive state of 156 patients followed one to five years. Arch. of Surg., 1944, 49, 180.

7. Levinson, J. E., Reiser, M. F., and Ferris, E. B., Jr., Variations in the blood pressure response to repeated administration of tetraethyl ammonium chloride. J. Clin. Invest., 1948, 27, 154. 